\title{
INVESTIGACIÓN
}

Recibido: 23/02/2021 --- Aceptado: 20/05/2021 --- Publicado: 03/01/2022

\section{CINE EN TORNO A LA EMERGENCIA CLIMÁTICA: UN PROBLEMA SIN RESPONSABLES NI SOLUCIONES}

\author{
Films about the climate emergency: a problem with no responsible neither \\ solutions
}

(iD) $\mathbb{R}^{6}$ David Vicente Torrico ${ }^{1}$. Universidad de Valladolid. España. david.vicente.torrico@uva.es

(8) $\mathbb{R}^{6}$ Nereida López Vidales. Universidad de Valladolid. España. nereida.lopez@uva.es

\section{Cómo citar el artículo:}

Vicente Torrico, D. y López Vidales, N. (2022). Cine en torno a la emergencia climática: un problema sin responsables ni soluciones. Vivat Academia. Revista de Comunicación, 155, 1-22. http://doi.org/10.15178/va.2022.155.e1311

El artículo se enmarca en la investigación titulada "Verdad y ética en las redes sociales. Percepciones e influencias educativas en jóvenes usuarios de Twitter, Instagram y YouTube (Internética)", financiada por la Convocatoria de Proyectos de I+D+i del Ministerio de Ciencia, Innovación y Universidades de 2019 (PID2019104689RB-100) y apoyada por el GIR Cultura digital, Innovación, Creatividad y Participación social en Comunicación: OCENDI, de la Universidad de Valladolid

\section{RESUMEN}

La industria del cine ha apostado por incluir la emergencia climática en sus relatos, configurando así un universo narrativo en el que el espectador negocia su identidad y sus propias actuaciones a través de las situaciones que aparecen en la pantalla. Mediante la aplicación de un análisis de contenido, nuestra investigación persigue desvelar las claves discursivas sobre las que se asienta el discurso cinematográfico, atendiendo a las variables temáticas representadas, a la construcción de los personajes y a los espacios recreados en las producciones exhibidas en las salas españolas entre los años 2000 y 2019. Los resultados revelan un tratamiento sesgado, tanto en la selección de variables como en el diseño de los personajes y escenarios, presentando un problema que afecta principalmente a la población occidental. En línea con lo señalado, el relato cinematográfico sobre la emergencia climática

\footnotetext{
${ }^{1}$ David Vicente Torrico: Investigador postdoctoral en la Universidad de Valladolid, donde ejerce como profesor en el área de Periodismo. Sus líneas de investigación se centran en la representación mediática del cambio climático y las catástrofes naturales y el estudio de la industria cinematográfica.
} 
presenta las mismas deficiencias narrativas que el discurso informativo, perdiendo así una valiosa oportunidad para transmitir valores sostenibles. Para evitar la inacción y la autocomplacencia entre el público, formulamos una serie de recomendaciones basadas en la contextualización y empoderamiento de la sociedad civil, a través del diseño de situaciones y personajes reconocibles para la audiencia.

PALABRAS CLAVE: Cine - Emergencia climática - Divulgación Eduentretenimiento - Educación ambiental - Narrativa - Película - Audiencia Guion

\section{ABSTRACT}

The film industry has opted to include the climatic emergency in its stories, thus configuring a narrative universe in which the spectator negotiates his identity and his own actions through the situations that appear on the screen. Through the application of a content analysis, our research aims to reveal the discursive keys on which the film discourse is based, paying attention to the thematic variables represented, the construction of the characters and the spaces recreated in the productions shown in Spanish cinemas between 2000 and 2019. The results reveal a biased treatment, both in the selection of variables and in the design of characters and settings, presenting a problem that mainly affects the Western population. In line with the above, the cinematic account of the climate emergency presents the same narrative deficiencies as the informative discourse, thus losing a valuable opportunity to transmit sustainable values. To avoid inaction and complacency among audiences, we make a series of recommendations based on contextualising and empowering civil society through the design of recognisable situations and characters for the audience.

KEYWORDS: Film - Climate emergency - Outreach - Edutainment - Environmental education - Narrative - Film - Audience - Story - Plot.

\section{CINEMA SOBRE A EMERGÊNCIA CLIMÁTICA: UM PROBLEMA SEM RESPONSÁVEIS NEM SOLUÇÕES}

\section{RESUMO}

A indústria do cinema tem investido em incluir a emergência climática nos seus relatos, configurando assim um universo narrativo onde o espectador negocia sua identidade e seus próprios atos através das situações que aparecem na tela. Através da aplicação de conteúdo, a nossa pesquisa procura desvendar as chaves discursivas sobre as quais se apresenta o discurso do cinema, atendendo às variáveis temáticas apresentadas, a construção de personagens, e os espaços recriados nas produções exibidas nas salas de cinema espanholas entre os anos 2000 e 2019. Os resultados revelam um tratamento tendencioso tanto na seleção das variáveis quanto no desenho dos personagens e cenários, apresentando um problema que afeta 
principalmente a população ocidental. De acordo com o descrito acima, o relato cinematográfico sobre a emergência climática apresenta as mesmas deficiências narrativas que o discurso informativo, perdendo assim uma oportunidade valiosa para transmitir os valores da sustentabilidade. Para evitar inação e a autocomplacência dentro do público, formulamos uma série de recomendações baseadas na contextualização e o empoderamento da sociedade civil, através da criação de situações e personagens reconhecíveis para a audiência.

\section{PALAVRAS CHAVE}

Cinema - Emergência climática - Divulgação - Eduentretenimento - Educação ambiental - Narrativa - Filme - Audiência - Roteiro

\section{INTRODUCCIÓN}

La sociedad del siglo XXI se enfrenta a la emergencia climática, un fenómeno científico que se ha incorporado con fuerza a las esferas política, mediática y social. No en vano, su inclusión entre los Objetivos de Desarrollo de la ONU, el incremento de la cobertura informativa (Fernández-Reyes, 2019) y la reciente movilización de la ciudadanía (Wahlström, Kocyba, De Vydt \& De Moor, 2019) han actuado como catalizadores del relato en torno a un problema cuya representación ha estado marcada tradicionalmente por más sombras que luces.

El periodismo científico ha tratado de proporcionar los argumentos y herramientas necesarias para facilitar la participación de los ciudadanos en el debate público sobre estas cuestiones. Sin embargo, en la práctica, las tensiones entre expertos y periodistas han provocado fisuras en el relato. A la disparidad de objetivos, criterios y procedimientos que guían su dinámica productiva (VicenteMariño y Vicente-Torrico, 2014), se suma el desinterés generalizado de los directores de los medios, que relegan la ciencia a un segundo plano, lo que se traduce en la falta de especialización de unos profesionales que reconocen no estar debidamente formados ni disponer de los medios necesarios para comprender y valorar las cuestiones científicas que tratan. La falta de sintonía entre los dos colectivos que participan del relato podría ser una de las razones de la escasa educación científica de la población, una realidad que, en el caso de la emergencia climática, se manifiesta en casi un tercio de los españoles según los últimos sondeos realizados por el Real Instituto Elcano (2019).

En el ámbito académico, la cobertura informativa del cambio climático es el tema medioambiental más recurrente entre los investigadores en ciencias sociales de las últimas décadas (Vicente-Mariño, 2011). Los autores señalan que se trata de un relato intermitente y cíclico (Carvalho, 2010), relegado a un segundo plano informativo (León-Anguiano, 2007), y vinculado a las catástrofes naturales y los encuentros políticos, por lo que prioriza el debate frente a la necesidad de actuar (Hulme, 2009). De este modo, el discurso mediático parece lejos de contribuir a formar una percepción adecuada de la gravedad de la situación. Pese a todo, el público sigue optando por los medios de comunicación como su principal ventana al mundo 
(López-Vidales; Gómez-Rubio y Vicente-Torrico, 2017), con una incidencia que alcanza hasta el 71\% en el caso de la emergencia climática (Díez-Nicolás, 2004).

Frente a las limitaciones inherentes al relato informativo, la educación ambiental se presenta como una propuesta formativa que persigue elevar el nivel de conocimiento general sobre la emergencia climática mediante la adquisición de valores y conductas responsables con el medio ambiente. Con más de medio siglo de historia, y amparada por la Organización de las Naciones Unidas para la Educación, la Ciencia y la Cultura (UNESCO) y por el Programa de las Naciones Unidas para el Medio Ambiente (PNUMA), la educación ambiental se dirige a todos los estratos sociales como un complemento de la formación reglada que debe desarrollarse a lo largo de la vida de las personas. Debido a su carácter informal, esta iniciativa pedagógica se apoya en el uso de técnicas como el eduentretenimiento (Tufte y Obregón, 2010), una disciplina orientada a la adquisición de creencias y modelos de comportamiento mediante el uso de formatos de ocio populares, lo que redunda en una mayor implicación por parte de los participantes. A partir de ideas simples, sencillas de comprender, el eduentretenimiento se apoya en los mecanismos de seducción propios de los soportes en los que se inserta, como la literatura, el cine, la publicidad o los videojuegos, para apelar más a la emoción que a la razón, generando así un flujo constante de estímulos que emergen de la cultura popular y que, en el caso de la emergencia climática, son clasificados bajo la etiqueta de ficción climática o Cli-Fi (Johns-Putra, 2016).

Aunque los primeros relatos con una temática medioambiental datan de mediados del siglo XX (Brereton, 2005), con el cine y la literatura como principal banco de pruebas, su presencia se ha multiplicado con la llegada del nuevo milenio, favoreciendo así la consolidación de este espacio narrativo. El éxito de estos formatos de entretenimiento residiría en la recreación de un entorno cotidiano, el desarrollo de una trama de fácil seguimiento, el uso de personajes reconocibles y la inclusión de una moraleja o enseñanza final (Jones \& Song, 2014), impulsados por el poder evocador de la narrativa y por la capacidad de seducción de la imagen (Sheppard, 2012).

Sin embargo, los estudios de recepción demuestran que los efectos producidos en el público a partir de este tipo de experiencias distan mucho de los resultados deseados, evidenciando con ello la dificultad para desarrollar una comunicación efectiva que permita combatir la crisis climática. Las experiencias vicarias, como señala Sakellari (2015), pueden generar un gran impacto en el corto plazo, pero no logran modificar el comportamiento de la audiencia. En esta misma línea se manifiestan Arendt y Matthes (2016), para los que el relato refuerza el interés de la audiencia ya familiarizada con el tema, pero no logra convencer a los espectadores neutrales. Entre las razones que podrían justificar esta baja sensibilización se encuentran el enfoque sensacionalista, la complejidad de las tramas, la falta de credibilidad de los personajes $\mathrm{y}$, por último, la repetición de discursos ya difundidos a través de los medios de comunicación (Vicente-Torrico, 2017). 
Por tanto, y a partir de estos antecedentes, proponemos analizar la representación cinematográfica de la emergencia climática y evaluar su grado de adecuación con respecto a los criterios que favorecen una adecuada concienciación social.

\section{OBJETIVOS}

El principal interés que persigue nuestra investigación es comprobar la validez del relato cinematográfico como herramienta educativa frente a la emergencia climática. Para ello, proponemos un estudio de las variables discursivas presentes en las películas que abordan esta realidad, a fin de elaborar un catálogo de recomendaciones que permitan impulsar la capacidad de concienciación en este tipo de narrativas.

Con este horizonte en mente, planteamos las siguientes preguntas de investigación:

1. ¿Qué imagen se proyecta acerca de las causas, consecuencias y posibles soluciones a la emergencia climática?

2. ¿Cuál es la presencia de los diferentes agentes sociales y qué funciones asumen de manera predominante?

3. ¿Qué escenarios aparecen representados en el retrato de la crisis climática?

La hipótesis de partida señala que el relato cinematográfico ofrece una visión sesgada y sensacionalista, alejada de los principios sugeridos por la narrativa ambiental para concienciar y fomentar la adopción de actitudes y comportamientos respetuosos con el medio ambiente, y favorece, por el contrario, la inacción y la autocomplacencia del público que acude a las salas y se expone ante este tipo de mensajes.

Esta afirmación se basa en el predominio de las consecuencias frente al análisis de las causas y la búsqueda de soluciones; en la adopción generalizada del punto de vista subjetivo de los afectados, en lugar de denunciar la participación de los responsables o resaltar las voces de alarma que parten de los colectivos expertos y organizaciones ecologistas; y en la recreación de escenarios que alejan al espectador del problema.

\section{METODOLOGÍA}

Para dar respuesta a las cuestiones planteadas y cumplir con el objetivo marcado de utilizar el cine como herramienta educativa, recurrimos a un protocolo de análisis diseñado de manera específica para el estudio de este tipo de materiales (VicenteTorrico, 2017), inspirado en la metodología sobre la que se sustentan los principales estudios en torno a la cobertura informativa de la crisis climática en los medios de comunicación tradicionales, ya señalados en el capítulo introductorio. 
Las características inherentes a la narrativa cinematográfica, con unas piezas de mayor duración y un lenguaje cargado de abstracción y simbolismo, exigen combinar acertadamente el carácter objetivo y sistemático del análisis de contenido con la capacidad de interpretación para describir no solo lo manifiesto en el mensaje, sino también lo latente (Krippendorff, 1990). De este modo, revelamos las características del relato atendiendo a su temática, a los personajes y a los escenarios en los que transcurre la acción. Para la detección de estos indicios se ha diseñado una ficha de codificación previa que combina el estudio cuantitativo y cualitativo, y que comprende un total de 32 campos distribuidos en tres categorías: el diseño de los personajes, las variables temáticas enunciadas y la configuración del escenario.

Tabla 1. Instrumento de análisis

\begin{tabular}{|c|c|c|c|c|c|c|}
\hline & Nombre & Eda & & Sexo & Colectivo & Rol \\
\hline $\begin{array}{c}\text { DISEÑO } \\
\text { PERSONAJES }\end{array}$ & & $\begin{array}{l}\text { Jov } \\
\text { Ad } \\
\text { An }\end{array}$ & $\begin{array}{l}\text { /a } \\
\mathrm{o} / \mathrm{a}\end{array}$ & $\begin{array}{l}\text { Masculino } \\
\text { Femenino }\end{array}$ & $\begin{array}{l}\text { Afectado/a } \\
\text { Ecologista } \\
\text { Empresario/a } \\
\text { Experto/a } \\
\text { Institucional } \\
\text { Periodista }\end{array}$ & $\begin{array}{l}\text { Protagonista } \\
\text { Antagonista } \\
\text { Secundario }\end{array}$ \\
\hline & Cau & & Con & ecuencias & Soluciones & Resolución \\
\hline $\begin{array}{l}\text { VARIABLES } \\
\text { ENUNCIADAS }\end{array}$ & $\begin{array}{l}\text { Antropog } \\
\text { Naturales }\end{array}$ & & $\begin{array}{l}\text { Hiel } \\
\text { Océc } \\
\text { Terr }\end{array}$ & & $\begin{array}{l}\text { Mitigación } \\
\text { Prevención } \\
\text { Adaptación }\end{array}$ & $\begin{array}{l}\text { Sí } \\
\text { No }\end{array}$ \\
\hline & Territorio & & & & Temporalidad & \\
\hline $\begin{array}{c}\text { DISEÑO } \\
\text { ESCENARIOS }\end{array}$ & $\begin{array}{l}\text { Global } \\
\text { Occidente } \\
\text { 3er mund } \\
\text { Otros (rec }\end{array}$ & & & & \begin{tabular}{|l} 
Pasado \\
Presente \\
Futuro
\end{tabular} & \\
\hline
\end{tabular}

Fuente: Elaboración propia

Los títulos que componen la muestra de análisis son las producciones cinematográficas exhibidas en las salas españolas entre los años 2000 y 2019. Para elaborar este corpus nos hemos apoyado en el listado elaborado por la International Environmental Communication Association (IECA) ${ }^{2}$, en portales de información cinematográfica especializada como $\mathrm{IMDB}^{3} \mathrm{y}$ en la base de datos proporcionada por el Ministerio de Educación, Cultura y Deporte (MECD) ${ }^{4}$, adoptando únicamente aquellos títulos cuya línea argumental abordase alguna de las cuestiones referidas a la emergencia climática, como son sus implicaciones en materia medioambiental, de

\footnotetext{
${ }^{2}$ https://theieca.org/filmography-nature-and-environmental-movies

${ }^{3}$ https://www.imdb.com

${ }^{4}$ http://www.culturaydeporte.gob.es/cultura/areas/cine/mc/catalogodecine/inicio.html
} 
salud, seguridad, economía, movilidad, vivienda, gestión de recursos o la búsqueda de energías alternativas, unas categorías temáticas que emergen de las recomendaciones formuladas por los investigadores que estudian la cobertura de la emergencia climática en los medios de comunicación tradicionales.

De este modo, hemos logrado avanzar desde una amplia recopilación de producciones cinematográficas hacia una muestra representativa y técnicamente operativa de la oferta audiovisual distribuida a lo largo de los últimos 20 años en el mercado español, gracias a la utilización de diferentes filtros conceptuales (criterio temporal, temático y circuito comercial) que han limitado de manera progresiva el número de resultados hasta alcanzar la muestra final, que queda conformada por un total de 92 títulos (ver anexo).

\section{RESULTADOS}

El análisis de los elementos que integran el discurso cinematográfico permite revelar, a través de su presencia o ausencia, los temas principales que definen la representación de la emergencia climática, así como las figuras que actúan como definidores primarios de esta realidad y los escenarios en los que se desarrollan las diferentes tramas.

\subsection{Estudio de las variables temáticas}

El estudio de las variables temáticas revela el predominio de los impactos, presentes en el $93 \%$ de las producciones $(n=86 / 92)$, por delante de la enunciación de las causas $(80 \%, \mathrm{n}=74 / 92)$ y la propuesta de soluciones $(79 \%, \mathrm{n}=73 / 92)$, como muestra la siguiente imagen. Esta circunstancia, ya de por sí relevante, será desgranada en los siguientes párrafos, ya que el relato cinematográfico incorpora de manera simultánea varias de las categorías temáticas analizadas en su guion.

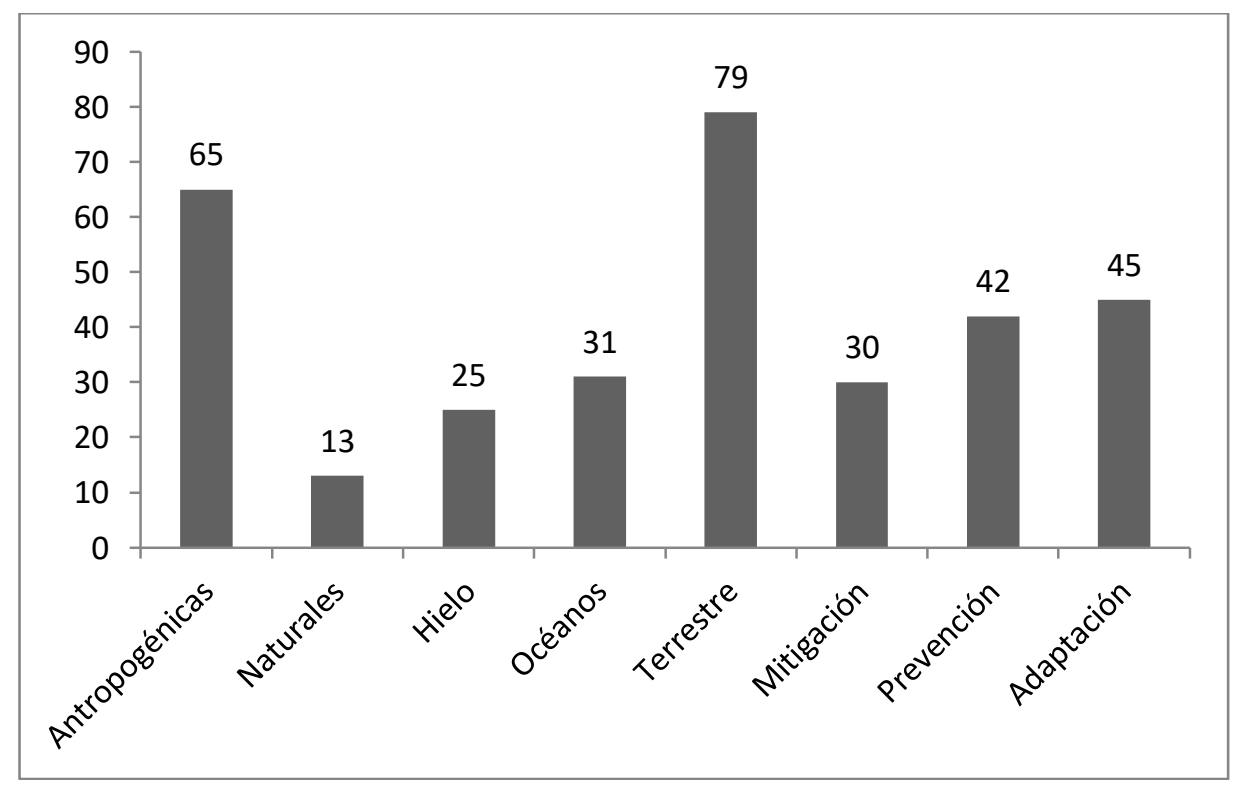

Figura 1. Películas en las que aparece cada categoría temática

Vivat Academia. Revista de Comunicación. 2022, nº 155, 1-22 
Fuente: Elaboración propia

El análisis de las causas aludidas en los diferentes proyectos aporta relevantes indicios en torno a la atribución de culpabilidad y la búsqueda de responsables. En este caso, y tomando en consideración las dos corrientes ideológicas que protagonizan el debate medioambiental, planteamos el estudio de la influencia antropogénica sobre el clima y el análisis de los agentes naturales que pueden afectar a la vida en el planeta. En este sentido, los resultados obtenidos demuestran el claro predominio de las causas asociadas a la intervención humana $(83 \%)$ frente a aquellas de origen natural $(17 \%)$.

Dentro de las causas antropogénicas, las variables que corresponden al acceso y uso de los recursos (42\%) se imponen a los problemas asociados a la gestión del suelo $(31 \%)$ y las emisiones derivadas del uso de combustibles fósiles, que aparecen en el $27 \%$ de los casos. De este modo, las actividades vinculadas a la gestión de residuos, al sector primario, a la deforestación o a la especulación urbanística tienen una mayor presencia en pantalla que aquellas relacionadas con la polución, ya sea a través de la industria, del transporte o del consumo energético en el ámbito doméstico.

En cuanto a las causas naturales, los responsables de las películas destacan como principales amenazas la radiación solar (35\%) y el impacto de meteoritos (29\%), por delante de las variaciones en el campo magnético (23\%) y la actividad volcánica (13\%). Aunque, como ya hemos señalado, la atribución de responsabilidades recae de manera mayoritaria sobre el ser humano, los factores naturales juegan un papel crucial en el relato cinematográfico, al ser el detonante de la trama en 8 de los títulos analizados.

Los agentes sociales que participan en la definición de las causas son un total de 255, y están encabezados por el grupo de las víctimas anónimas de la emergencia climática (35\%), superando ampliamente al colectivo formado por expertos $(22 \%)$. Mientras los primeros señalan al ser humano como responsable del problema (86\%), el discurso científico presenta una visión holística de la crisis medioambiental, ya que dedican la cuarta parte de sus intervenciones a analizar la influencia de los agentes naturales sobre el clima. El sector empresarial, responsable de esta situación a ojos de la opinión pública, aparece en tercer lugar (18\%), y su relato gira principalmente en torno a las actividades relacionadas con el acceso y uso del suelo.

Las películas que abordan las causas de la emergencia climática presentan este problema desde una perspectiva global (47\%). Los países occidentales (27\%) ocupan la segunda posición, por delante de la recreación de territorios alternativos (19\%). En consecuencia, el relato cinematográfico exime de toda culpa a las naciones en vías de desarrollo, que apenas albergan el 7\% de los títulos analizados. 


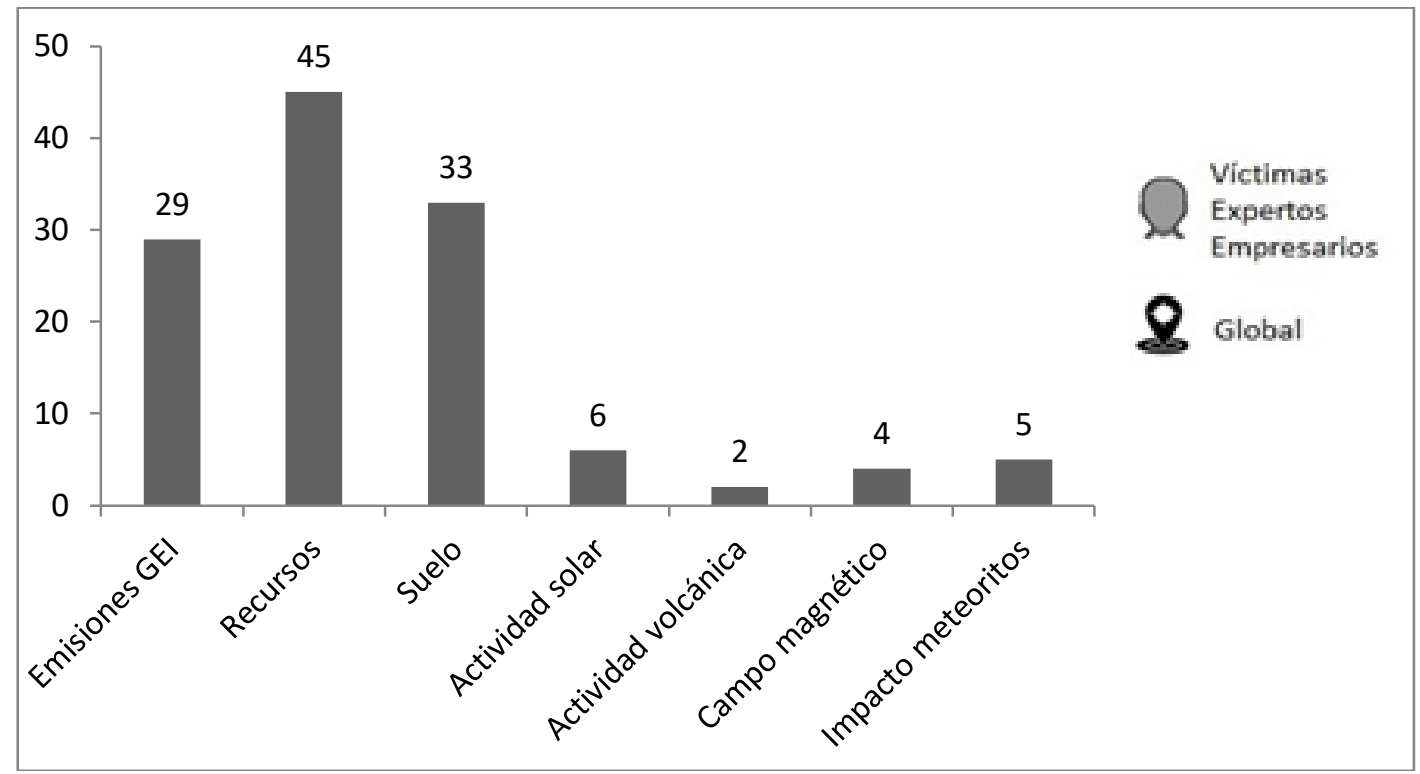

Figura 2. Representación cinematográfica de las causas de la crisis climática Fuente: Elaboración propia

El estudio de las consecuencias contribuye a la definición de la emergencia climática, estableciendo una relación causa-efecto en la representación llevada a cabo por la industria del cine. Este apartado engloba los impactos producidos sobre la superficie helada, los efectos generados en la superficie oceánica y las consecuencias derivadas de la crisis climática sobre la superficie terrestre.

Los resultados obtenidos demuestran que el discurso cinematográfico se decanta por mostrar los efectos producidos sobre el territorio continental (72\%), en línea con la percepción antropocéntrica del entorno natural. En segundo lugar, y a una notable distancia, se encuentran los impactos generados en la superficie oceánica (16\%), que logran obtener una mayor cuota de pantalla que los territorios helados (12\%).

A nivel específico, los efectos sobre la superficie terrestre se escriben en términos de acción y drama, y, en menor medida, a través de los títulos de animación. Por ello, las variables más recurrentes van a estar vinculadas al sufrimiento de los personajes, ya sea a través de la pérdida de biodiversidad (25\%), la escasez de recursos (20\%), los conflictos y movimientos migratorios (14\%) y las afecciones sobre la salud (9\%). En el lado contrario, las consecuencias relacionadas con los cambios experimentados por los parámetros naturales, tales como los cambios de temperatura (13\%), de los ciclos de precipitaciones $(11 \%)$ y la pérdida de rendimiento de los cultivos $(8 \%)$ reciben una menor atención, y su presencia queda supeditada a las producciones documentales.

En el caso de las variables relacionadas con los océanos, el relato cinematográfico establece una clara división entre los fenómenos evolutivos y los disruptivos. De este modo, el primer grupo queda conformado por los cambios en las corrientes oceánicas (7\%) y en la composición química del agua (9\%), en un relato técnico que encuentra acomodo en el género documental. En el otro lado, la elevación del nivel del mar $(29 \%)$ y la formación de fenómenos naturales extremos (55\%) suponen importantes 
giros narrativos, y se convierten en elementos fundamentales para el drama y la acción.

El descenso de la superficie helada es uno de los símbolos de la emergencia climática, aunque para la industria del cine apenas tiene visibilidad. Mediante un planteamiento didáctico, el relato documental describe el efecto albedo (15\%) y las consecuencias ligadas al deshielo de los glaciares (18\%), mientras que el retroceso de los polos (67\%), que es la variable dominante en este apartado, aparece en todos los tipos de géneros.

Atendiendo a las características de los personajes, el grupo que protagoniza el relato de las consecuencias es el de las víctimas (37\%). A continuación aparecen los colectivos de expertos (24\%) e instituciones (10\%), que, ya sea en el documental o en la ficción, protagonizan acalorados enfrentamientos sobre los efectos de la emergencia climática. El atractivo visual de las catástrofes naturales facilita la inclusión del gremio periodístico $(8 \%)$, que encuentra su espacio como definidor primario de la amenaza medioambiental desde el mismo lugar de los hechos.

En términos geográficos, los impactos de la emergencia climática se muestran en clave global $(51 \%)$, impulsado por la presencia de los problemas vinculados a las superficies heladas y oceánicas. La variable occidental obtiene también una elevada visibilidad (25\%), y destaca en la representación de los problemas sobre la superficie terrestre. Las consecuencias que sufren los países en vías de desarrollo vuelven a ser invisibilizadas (7\%), quedando incluso por detrás de los escenarios recreados $(17 \%)$.

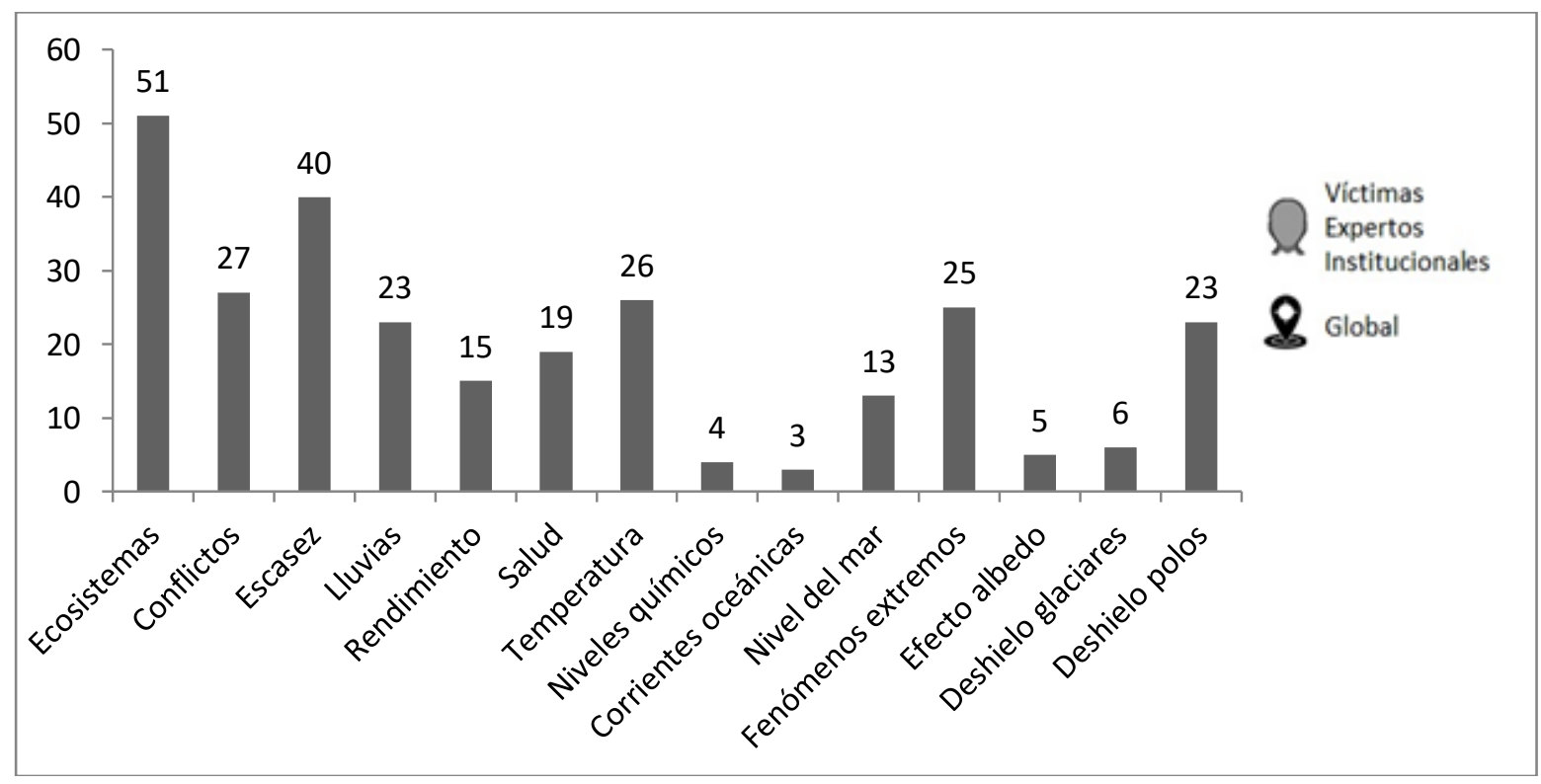

Figura 3. Representación cinematográfica de las consecuencias de la crisis climática

Fuente: Elaboración propia

La adopción de un tratamiento adecuado para combatir los efectos de un problema requiere partir de una correcta definición del mismo. Una vez analizadas 
las dos primeras componentes del mensaje cinematográfico, concluimos nuestro análisis de las variables temáticas mediante el estudio de las medidas reflejadas por el cine.

Según los datos obtenidos, las propuestas para mitigar el impacto de la crisis climática obtienen la menor representación $(27 \%)$ en el estudio temático, en un discurso que favorece a las medidas de prevención (43\%) y la adaptación a las nuevas circunstancias del entorno (30\%).

Entre las soluciones mitigadoras, encaminadas a erradicar la situación de emergencia climática, destacan las energías renovables (25\%) y la reforestación de los bosques $(23 \%)$, ambas presentes en todos los géneros cinematográficos. La geoingeniería $(23 \%)$ cobra protagonismo en el cine de ficción, a través de la recreación de diversos experimentos que constituyen el eje del relato. El resto de medidas, con un carácter más técnico, como la reducción de gases contaminantes $(15 \%)$, su captura en sumideros $(8 \%)$ o la negociación de derechos de emisión $(6 \%)$, aparecen vinculadas al relato documental.

Las medidas encaminadas a la prevención de daños ante unos impactos ya inevitables podrían dividirse entre oficiales y sociales. Entre las primeras, dependientes de los poderes públicos, se encuentran la protección del entorno y sus especies $(23 \%)$, la adopción de acuerdos políticos $(16 \%)$, la educación ambiental $(15 \%)$, la inversión en infraestructuras adaptadas a las nuevas condiciones (7\%), la puesta en marcha de mecanismos de control y denuncia $(7 \%)$ y el diseño de un sistema de alerta temprana (6\%). El segundo grupo tiene un carácter reivindicativo y engloba las movilizaciones ecologistas (26\%), que suponen la variable más recurrente en este apartado temático.

En cuanto a las propuestas adaptativas, el relato cinematográfico se decanta por la evacuación (61\%), una variable que destaca en los guiones de ficción y animación. Las soluciones destinadas a favorecer la convivencia con los nuevos patrones climáticos, como la eficiencia tecnológica (16\%), el reciclaje (13\%) y la recuperación de los entornos dañados $(10 \%)$ reciben una atención inferior, a pesar de que su presencia ha sido detectada en todos los géneros cinematográficos analizados.

Las fuentes que participan en el relato sobre las soluciones aparecen encabezadas por el colectivo de víctimas $(24 \%)$, debido a su elevada presencia en el apartado de las evacuaciones. A escasa distancia se sitúa el grupo de expertos (23\%), cuya aportación al retrato cinematográfico se centra en las soluciones mitigadoras. En tercera posición encontramos a los representantes públicos $(14 \%)$, con un peso similar a los colectivos ecologista y empresarial (13\%), que se benefician de la agitación social y del progreso tecnológico respectivamente para ganar visibilidad.

Los escenarios en los que se desarrollan las tramas relacionadas con las soluciones vuelven a situar al enfoque global en primer lugar (54\%), doblando en número a los relatos que se desarrollan en los países occidentales $(27 \%)$. Lo relevante de estos 
resultados es que, una vez que el impacto es inevitable, la acción se desarrolla en los territorios recreados $(14 \%)$, dibujando un horizonte complicado. Este hecho tiene una lectura final, y es que los países en vías de desarrollo (5\%) apenas participan en la reconstrucción del planeta, y dependen de la protección que les brinden las grandes potencias mundiales.

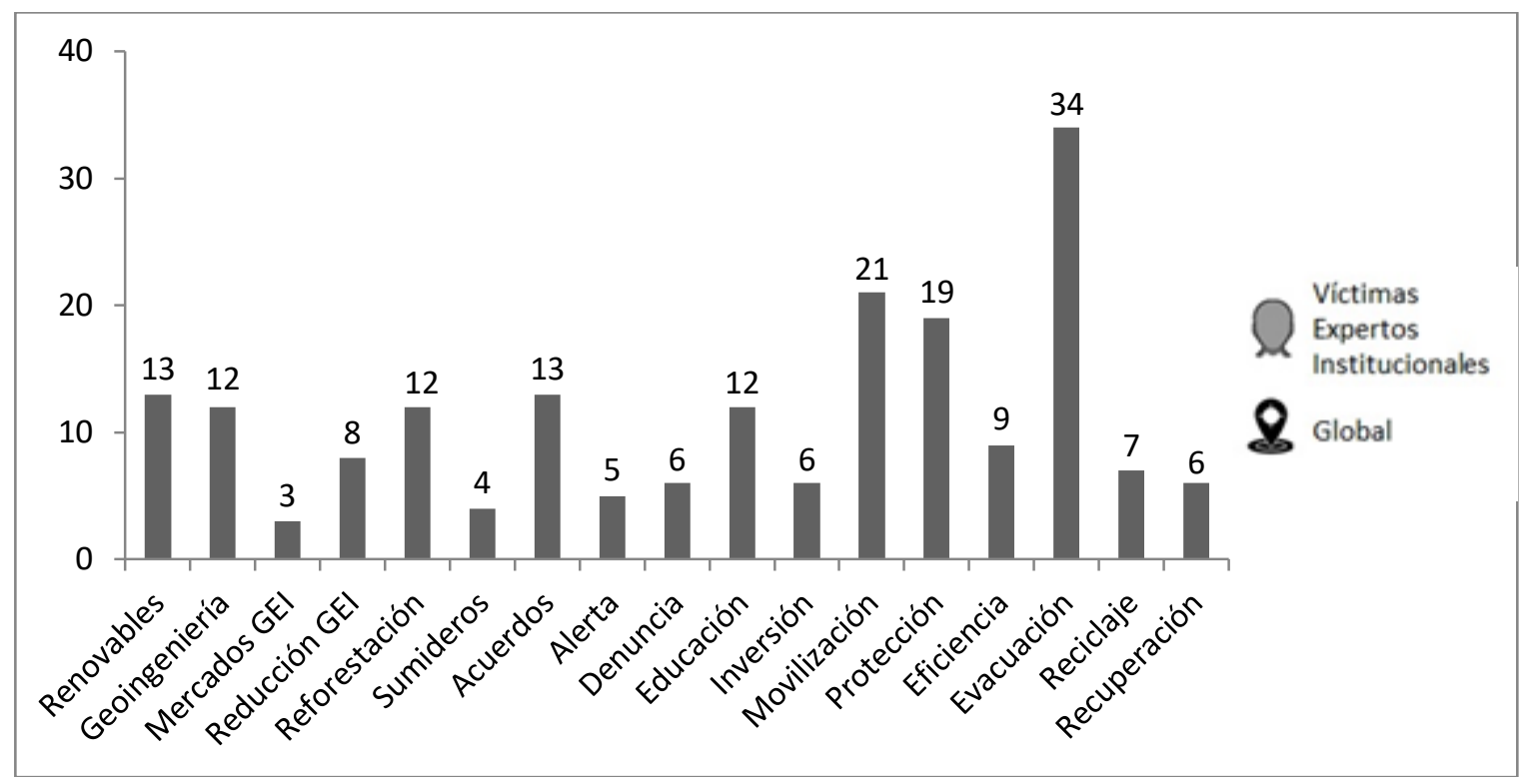

Figura 4. Representación cinematográfica de las soluciones a la crisis climática Fuente: Elaboración propia

Como cierre del apartado dedicado a las soluciones hemos analizado el grado de efectividad de las propuestas que aparecen en el relato cinematográfico. El desenlace de las diferentes tramas señala que solo en el $42 \%$ de los casos se ha alcanzado una resolución satisfactoria, mientras que en el 58\% restante los esfuerzos realizados por los personajes para revertir la situación resultan estériles.

En este banco de pruebas virtuales que es el cine, los primeros relatos apuestan por una resolución positiva del problema, y confieren a sus personajes la capacidad de devolver al planeta a su estado de equilibrio. Sin embargo, a partir del estreno de Una verdad incómoda (2006) se adopta un enfoque más crítico, en el que las catástrofes resultan tan complejas que no siempre se puede combatir el problema. En este periodo, el género cinematográfico juega un papel fundamental, ya que la animación va a convertirse en el último reducto para la humanidad, a través del clásico final feliz. Por el contrario, el cine documental, dirigido a un público adulto y crítico, muestra una concepción pesimista sobre el devenir del planeta, a pesar de las medidas propuestas.

De entre las soluciones señaladas, la prevención obtiene un mayor índice de éxito (53\%), por delante de las medidas adaptativas (31\%) y las mitigadoras $(16 \%)$, en un discurso que parece plantear que la emergencia climática es ya un hecho inevitable. A nivel específico, las propuestas que gozan de una mayor aceptación por parte de los responsables de las producciones serían la reforestación, la geoingeniería, la 
Vicente Torrico, D. y López Vidales, N.

Cine en torno a la emergencia climática: un problema sin responsables ni soluciones

inversión económica, la movilización social, la protección del entorno y, aunque con un margen exiguo, la evacuación de la población hacia territorios seguros.

\subsection{Estudio de los personajes que intervienen en el relato}

El siguiente apartado a considerar en nuestro análisis de contenido es el estudio de los personajes que participan en la representación cinematográfica de la crisis climática, ya que estas figuras inciden en los mecanismos de identificación que se generan en el espectador y juegan un papel fundamental a la hora de definir el objeto de estudio.

Aunque los resultados de las variables temáticas presentan algunos indicios relevantes en torno al rol de los personajes, hemos creído oportuno recopilar en un epígrafe propio todos aquellos datos que permitan avanzar en el análisis de estos elementos.

El primer rasgo diferencial que hemos analizado es la adscripción de los personajes a un determinado colectivo, generalmente vinculado a su profesión. Así, comprobamos que el grupo más numeroso está formado por las víctimas de la crisis climática (38\%), muy por delante del colectivo de expertos y de los representantes públicos, que alcanzan una representación del 15\%. El sector empresarial engloba al $13 \%$ de los personajes, mientras que periodistas y ecologistas reducen su presencia hasta el $8 \%$. El porcentaje restante correspondería a los narradores de la acción en off $(3 \%)$.

Si analizamos los datos demográficos del elenco que participa en la representación de la emergencia climática en el cine, comprobamos que casi dos terceras partes de estas figuras corresponden al perfil del varón de mediana edad. En consecuencia, los papeles femeninos (22\%) o las figuras más jóvenes del elenco (9\%) pasan desapercibidas. Además de su escaso número, estas figuras van a jugar un papel secundario en el relato, generalmente asociado a víctimas o a movimientos ecologistas.

En último lugar, debemos reseñar la existencia de voces discrepantes en el relato, en lo que se ha dado a conocer como negacionistas del clima. Estas figuras, que cuestionan el consenso científico frente a la emergencia climática, promueven una definición alternativa del problema a través de roles antagónicos y encuadres declarativos. Las alusiones religiosas $(1 \%)$, que equiparan el conocimiento científico con la existencia de una fuerza divina, presentan un alto nivel de arraigo entre las víctimas de los países en vías de desarrollo. Los escépticos del clima (9\%), impulsados por lobbies cuya actividad está relacionada con la huella ecológica, aparecen ubicados en los países occidentales y, a pesar de su redención final, desempeñan el papel de antagonistas durante la mayor parte del relato. También es posible encontrarlos en el cine documental, a través de las declaraciones de archivo. 


\subsection{Análisis de los escenarios / territorios}

La delimitación del espacio en el que transcurre la acción supone el último apartado de nuestro análisis, y aporta una lectura adicional a las variables estudiadas hasta ahora. Como se ha señalado en el capítulo introductorio, la emergencia climática se define en términos de distanciamiento, tanto físico como temporal, por lo que el estudio de estas coordenadas permite ahondar en la representación del fenómeno.

A lo largo de nuestro estudio hemos detectado un total de 364 escenarios diferentes, de los cuales el 37\% corresponden a territorio occidental, frente al $9 \%$ de los países en vías de desarrollo y el 19\% de los lugares recreados. La variable global, cuando el relato se desarrolla de forma simultánea en varios espacios a la vez, suma el $35 \%$ del total.

A nivel específico, comprobamos que el principal foco de atención es Estados Unidos, cuya presencia en el discurso cinematográfico supone un $29 \%$ de los casos, y suma más impactos que todos los países en vías de desarrollo juntos. El factor país aplicado a la muestra incide en este apartado, al situar a España como el segundo territorio más relevante $(9 \%)$, por delante de países más grandes como China $(7 \%)$, Reino Unido (6\%) Brasil (4\%) o la India (3\%). En el lado contrario, el continente africano y las islas del Pacífico tienen una presencia testimonial a lo largo de la investigación.

Este tratamiento desigual también se hace patente en el análisis de las variables que se representan en cada escenario. De este modo, el origen antropogénico de la crisis climática se asocia en dos de cada tres casos a las sociedades avanzadas, mientras que en el abordaje de las causas naturales juegan un importante papel los escenarios recreados $(38 \%)$, desde un futuro distópico en el que la humanidad ha colapsado. La alteración de los patrones naturales del clima es abordada en clave internacional, mientras que las consecuencias más disruptivas, como la subida del nivel del mar o los grandes fenómenos naturales, golpean a occidente. Los efectos de tipo evolutivo, como la escasez de recursos y los conflictos que esto provoca, aparecen en los países en vías de desarrollo. Las zonas más pobres del planeta apenas participan en las medidas para combatir la amenaza, y limitan su capacidad de reacción a la evacuación. Las sociedades modernas concentran el grueso de soluciones, ya sean mitigadoras, preventivas o reactivas, aunque el relato cinematográfico esconde un último recurso, la recuperación del planeta en un tiempo futuro, desde un escenario recreado $(20 \%)$.

\section{DISCUSIÓN}

Los resultados expuestos confirman que los impactos de la crisis climática suponen la principal vía de entrada a la realidad de este fenómeno en el relato cinematográfico. Estos indicios coinciden con la cobertura realizada por los medios 
de comunicación tradicionales, ya sea en prensa (Young \& Dugas, 2011) o televisión (Djerf-Pierre, 2012).

La representación de las causas de la emergencia climática en el cine muestra las mismas carencias que su cobertura periodística. Como apunta Gunster (2011), el discurso en torno a las causas presenta un abordaje muy superficial, sin profundizar en el modo en que la población contribuye a este problema con su dieta, medios de transporte, ocio o vivienda. Además, rara vez se establece una conexión directa con las consecuencias ni se señala a los responsables (Brereton, 2005).

La ausencia generalizada de comportamientos ejemplares y soluciones al alcance del público refleja una tendencia que también ha sido detectada en el discurso elaborado por los medios de comunicación. De este modo, autores como Shwom, Dan y Dietz (2008) vinculan la baja percepción de autosuficiencia e implicación de la población a la reducida presencia de medidas asequibles para el público en el relato diario.

El diseño de los personajes cinematográficos contradice el reparto de tiempos registrado en los medios de comunicación tradicionales, donde instituciones y expertos protagonizan un relato en el que las víctimas aparecen en último lugar (Young \& Dugas, 2011). Sin embargo, nuestros resultados presentan ciertas similitudes con el periodismo de catástrofes (Rodríguez y Odriozola-Farré, 2012).

Además del colectivo al que pertenecen los personajes, el estudio demográfico de los mismos reproduce el modelo de representación de los medios de comunicación, donde las mujeres y los jóvenes, que son los grupos sociales más implicados en la defensa del medio ambiente, reciben una menor atención periodística (Corner et al., 2015).

Los escenarios en los que se desarrollan las tramas analizadas se concentran en el mundo occidental, dejando al margen del relato aquellos territorios más afectados por la crisis climática, como los países en vías de desarrollo y las islas del Pacífico. Brereton (2005), en su repaso a la representación de los problemas medioambientales durante el siglo XX, define Estados Unidos como quintaesencia del postmodernismo y el lugar propicio para el desarrollo de todo tipo de catástrofes, una idea que comparte González Alcaraz (2014) en su estudio sobre la prensa latinoamericana.

\section{CONCLUSIONES}

De acuerdo con los objetivos marcados en la investigación, a lo largo del capítulo de resultados hemos presentado la relación de variables temáticas que determinan la representación cinematográfica de la emergencia climática, mediante la aplicación de un diseño metodológico basado en el análisis de contenido. De este modo, y tras haber analizado un total de 92 películas, procedemos a evaluar la validez del relato cinematográfico como instrumento para la concienciación de la población. 
En el apartado temático, nuestro punto de partida aventuraba un relato sesgado, con un marcado predominio de los efectos más destructivos frente al análisis crítico de las causas, la búsqueda de culpables y la promoción de soluciones. Los resultados expuestos confirman que los impactos de la crisis climática aparecen representados en el 93\% de las películas, y son enunciados por tres de cada cuatro personajes, por lo que suponen la principal vía de entrada a la realidad del fenómeno. Dentro de esta categoría, el relato se centra en las variables que representan una amenaza disruptiva. Por el contrario, los efectos evolutivos, vinculados a la alteración de los patrones naturales, aparecen de forma puntual en el relato documental.

La segunda categoría temática por número de apariciones corresponde a las causas, que están presentes en el $80 \%$ de los títulos analizados. El hecho de que varios de los relatos comiencen en un futuro distópico, sin hacer referencia a los motivos que llevaron a dicha situación, contribuye de manera notable a la descontextualización del problema. Aunque el relato cinematográfico otorga un claro predominio a los factores humanos, cuya presencia multiplica por cinco a los argumentos sobre el origen natural del fenómeno, su carácter autocrítico se reduce al género documental, y presenta un retrato edulcorado del actual sistema de producción y consumo.

El asunto con una menor representación en el discurso cinematográfico corresponde a la propuesta de soluciones, presentes en el 79\% de las obras. De hecho, si tenemos en cuenta el índice de efectividad de estas medidas, comprobamos que más de la mitad de los títulos concluye sin haber aplicado ningún tipo de solución, lo que incapacita al público para adoptar un patrón de conducta responsable, fomentando con ello la inacción y la autocomplacencia. A esta idea contribuye la escasa presencia de los pequeños gestos, como el reciclaje o la eficiencia energética, medidas al alcance de un público que podría sentirse empoderado para actuar a través de este modelo.

Por lo tanto, y desde el punto de vista temático, hemos podido confirmar que el relato cinematográfico tiende a priorizar los aspectos más espectaculares del problema, en una representación de la crisis climática que peca de sensacionalista.

La segunda pregunta de investigación analiza la presencia y funciones de los distintos colectivos que participan en el espacio público, y los resultados han permitido confirmar que la cámara adopta principalmente el punto de vista de las víctimas. Con una presencia del 38\%, su perspectiva resulta perfecta para aportar dramatismo al guion, ya que expone a un personaje vulnerable ante una amenaza de la que, a priori, no es responsable. El grupo de expertos, socialmente reconocido como la fuente más fiable para abordar esta situación, supone el 15\% de la muestra, y su presencia se divide entre las declaraciones divulgativas de los documentales y el papel de superhéroes en el cine de ficción, en un retrato distorsionado de este colectivo. Por el contrario, los dos grupos que a priori consideramos responsables del deterioro del planeta, el sector empresarial y los representantes públicos, confirman su papel antagónico a través de un perfil caricaturesco o del montaje por contraste. 
Además de lo indicado, la construcción de los personajes presenta dos importantes factores de mejora desde el punto de vista de la concienciación medioambiental: de un lado, las figuras antropomórficas (19\%) generan empatía entre el público, pero limitan la identificación con ellos; del otro, el perfil del personaje analizado responde al varón occidental de mediana edad, por lo que excluye a jóvenes, mujeres y otras etnias. Por ello, podemos concluir que desde el punto de vista de los personajes también se presenta un relato sensacionalista y discriminatorio.

El tercer punto de nuestro análisis remite a la definición del espacio en el que se desarrolla la acción. A partir de los resultados obtenidos, comprobamos que el cine recrea un territorio fácilmente identificable para el público occidental. Esta cercanía podría reducir el distanciamiento psicológico frente al problema, al situar en una posición de riesgo el entorno más inmediato, las posesiones y los seres queridos de la audiencia. Sin embargo, el carácter eurocéntrico de la narración, con occidente como epicentro de la emergencia climática, deja fuera del foco los territorios más afectados, como los países en vías de desarrollo y las islas del Pacífico. Estos escenarios, habitualmente situados en los márgenes del relato, quedan fuera de la toma de decisiones y de la puesta en marcha de soluciones para combatir el problema.

En definitiva, las conclusiones obtenidas presentan un relato cinematográfico con luces y sombras, en las que hemos podido confirmar nuestras hipótesis de partida. A tenor de los resultados expuestos podemos señalar que el cine realiza un acercamiento sensacionalista, tanto desde el punto de vista temático como del diseño de los personajes, pues fomenta el enfoque humano, disruptivo y dramático del fenómeno. Por estos motivos, no cabe esperar un mayor compromiso en la lucha contra la emergencia climática por parte del espectador respecto a la audiencia de los medios tradicionales, ya que ambas narrativas presentan las mismas carencias.

\section{RECOMENDACIONES}

En línea con el último de los objetivos marcados, procedemos a ofrecer una serie de recomendaciones que contribuirían a potenciar la capacidad de concienciación del relato medioambiental. Este punto, no obstante, debe ser considerado como la propuesta de una nueva línea de trabajo y nunca como un planteamiento prescriptivo, ya que nuestro proyecto se plantea desde el más estricto plano analítico del investigador, para lo que nos hemos servido de las carencias narrativas expuestas en la literatura consultada. Las posibles mejoras a realizar en los relatos del futuro deberían incluir: 1) una mayor contextualización, dotando de más presencia en la trama a las causas que originaron el problema; 2) un mensaje capacitador, en el que se muestren los pequeños gestos que permitan al espectador contribuir a la lucha contra la crisis climática; 3) una mayor presencia de expertos $y$, especialmente, de grupos ecologistas, cuyo discurso y ejemplo sirvan a los intereses de la labor de concienciación; 4) un proceso de empoderamiento femenino, juvenil y étnico, ya que en el discurso actual se excluye a la mayoría de la población mundial, dificultando 
así su identificación como agentes activos; y 5) mostrar a personas, frente a la presencia de seres y animales humanizados, ya que este tipo de figuras dificultan la implicación de la audiencia.

\section{REFERENCIAS}

Arendt, F. \& Matthes, J. (2016). Nature documentaries, connectedness to nature, and pro-environmental behavior. Environmental Communication, 10(4), 453-472. https://doi.org/10.1080/17524032.2014.993415

Brereton, P. (2005). Hollywood utopia: Ecology in contemporary American cinema. Intellect Books.

Carvalho, A. (2010). Media(ted) discourses and climate change: a focus on political subjectivity and (dis)engagement. Wiley Interdisciplinary Reviews: Climate Change, 1(2), 172-179. https://doi.org/10.1002/wcc.13

Corner, A., Roberts, O., Chiari, S., Völler, S., Mayrhuber, E. S., Mandl, S. \& Monson, K. (2015). How do young people engage with climate change? The role of knowledge, values, message framing, and trusted communicators. Wiley Interdisciplinary Reviews: Climate Change, 6(5), 523-534. https://doi.org/10.1002/wcc.353

Díez-Nicolás, J. (2004). El dilema de la supervivencia: los españoles ante el medio ambiente. Obra Social Caja Madrid.

Djerf-Pierre, M. (2012). When attention drives attention: Issue dynamics in environmental news reporting over five decades. European Journal of Communication, 27(3), 291-304. https:// doi.org/10.1177/0267323112450820

Fernández-Reyes, R. (2019). Sigue la tendencia de récords: el mes de octubre de mayor cobertura histórica. Comunica Cambio Climático. https://comunicacambioclimatico.files.wordpress.com/2019/11/11.-resumenoctubre-2019.pdf

González-Alcaraz, L. (2014). El framing como legitimación de la política climática. Encuadres del cambio climático en la prensa argentina y brasileña durante las conferencias de Doha y Varsovia. Anuario Electrónico de Estudios en Comunicación Social "Disertaciones", 7(1), 224-259.

Gunster, S. (2011). Covering Copenhagen: Climate politics in BC media. Canadian Journal of Communication, 36(3), 477-502. 
Cine en torno a la emergencia climática: un problema sin responsables ni soluciones

Hulme, M. (2009). Why we disagree about climate change: Understanding controversy, inaction and opportunity. Cambridge University Press.

Johns-Putra, A. (2016). Climate change in literature and literary studies: From cli-fi, climate change theater and ecopoetry to ecocriticism and climate change criticism. Wiley Interdisciplinary Reviews: Climate Change, 7(2), 266-282. https://doi.org/10.1002/wcc.385

Jones, M. D. \& Song, G. (2014). Making sense of climate change: How story frames shape cognition. Political Psychology, 35(4), 447-476. https://doi.org/10.1111/pops.12057

Krippendorff, K. (1990). Metodología del Análisis de Contenido. Teoría y Práctica. Paidós.

León-Anguiano, B. (2007). El medio ambiente en las televisiones españolas. Un análisis de contenido de los informativos nacionales. En: F. R. Contreras (dir.), Cultura verde, ecología, cultura y comunicación (361-373). Consejería de Medio Ambiente.

López-Vidales, N., Gómez-Rubio, L. y Vicente-Torrico, D. (2017). La información territorial en la radio y la televisión de ámbito nacional en España y su contribución a la imagen de las comunidades autónomas. Revista Latina de Comunicación Social, 72, 649-666. https://doi.org/10.4185/RLCS-2017-1184

Real Instituto Elcano (2019). Los españoles ante el cambio climático. Apoyo ciudadano a los elementos, instrumentos y procesos de una Ley de Cambio Climático y Transición Energética. https://blog.realinstitutoelcano.org/los-espanoles-ante-el-cambioclimatico/

Rodríguez, P. y Odriozola-Farré, B. (2012). Catástrofes y periodismo: el relato, los escenarios, las interacciones y las necesidades prácticas y psicológicas de todos los implicados. Estudios sobre el mensaje periodístico, 18(2), 577-594. https://doi.org/10.5209/rev_ESMP.2012.v18.n2.41033

Sakellari, M. (2015). Cinematic climate change, a promising perspective on climate change communication. Public Understanding of Science, 24(7), 827-841. https://doi.org/10.1177/0963662514537028

Sheppard, S. (2012). Visualizing climate change: a guide to visual communication of climate change and developing local solutions. Routledge.

Shwom, R., Dan, A. \& Dietz, T. (2008). The effects of information and state of residence on climate change policy preferences. Climatic Change, 90(4), 343-358. https://doi.org/10.1007/s10584-008-9428-7 
Tufte, T. y Obregón, R. (2010). Edu-entretenimiento y cambio social: hacia una nueva agenda conceptual. Son de Tambora, 269.

Vicente-Mariño, M. (2011). La expansión de la investigación sobre comunicación medioambiental: ¿otra consecuencia más del cambio climático? En: S. ÁlvarezCantalapiedra (coord.). Convivir para perdurar. Conflictos ecosociales y sabidurías ecológicas (371-387). Icaria.

Vicente-Mariño, M. y Vicente-Torrico, D. (2014). Presencia y funciones del discurso científico en la cobertura informativa y cinematográfica del cambio climático. Prisma Social. Revista de Investigación Social, 12, 120-152.

Vicente-Torrico, D. (2017). El cine como herramienta educativa para explicar el cambio climático. Una propuesta experimental. En: III Congreso Internacional de Educación Mediática y Competencia Digital, 817-827.

Wahlström, M., Kocyba, P., De Vydt, M. \& De Moor, J. (2019). Protest for a future: Composition, mobilization and motives of the participants in Fridays For Future climate protests on 15 March, 2019 in 13 European cities. https://gup.ub.gu.se/publication/283193

Young, N. \& Dugas, E. (2011). Representations of climate change in Canadian national print media: The banalization of global warming. Canadian Review of Sociology, 48(1), 1-22. https:// doi.org/10.1111/j.1755-618X.2011.01247.x

\section{AUTOR/ES:}

\section{David Vicente Torrico:}

Investigador postdoctoral en la Universidad de Valladolid, donde ejerce como profesor en el área de Periodismo. Sus líneas de investigación se centran en la representación mediática del cambio climático y las catástrofes naturales y el estudio de la industria cinematográfica. Forma parte del grupo de investigación reconocido Ocendi (Observatorio del Ocio y el Entretenimiento Digital) y colabora en los talleres medioambientales de Climántica.

david.vicente.torrico@uva.es

Orcid ID: https:/ / orcid.org/0000-0003-0379-6086

Google Scholar: https://scholar.google.es/citations?user=3DMiJ-0AAAAJ\&hl=es

ResearchGate: https://www.researchgate.net/scientific-contributions/2165115907David-Vicente-Torrico

Scopus ID: $\underline{\text { https: / / www.scopus.com/authid/ detail.uri?authorId }=56340374000}$

Academia.edu: https://uva-es.academia.edu/DavidVicenteTorrico

\section{Nereida López Vidales:}

Profesora Titular del Grado en Periodismo de la Universidad de Valladolid. Es Doctora en Ciencias Políticas y Sociología, Periodista, Politóloga y Máster en 
Producción Radiofónica. Ha trabajado en varios medios de comunicación y compagina la docencia universitaria con la dirección del Observatorio del ocio y el entretenimiento digital (GIR OCENDI), la dirección de Radio UVa y las Coordinaciones del Grado en Periodismo y el Programa de Doctorado ELLCOM (UVa). Sus líneas de investigación son la tecnología audiovisual, la radio y la televisión, la evolución de los perfiles profesionales, jóvenes y tendencias de consumo mediático, y la creación de nuevos contenidos. Ha publicado varias decenas de artículos científicos y once libros.

nereida.lopez@uva.es

Orcid ID: https:// orcid.org/0000-0002-6960-6129

Google Scholar: https://scholar.google.es/citations?user=d-h-uasAAAAJ\&hl=es

ResearchGate: https://www.researchgate.net/profile/Nereida_Lopez_Vidales

Scopus ID: https: / / www.scopus.com/authid/detail.uri?authorId=56009001000

Academia.edu: https://independent.academia.edu/nereidalopez 


\section{ANEXOS}

Anexo 1: Listado de películas que componen la muestra de análisis

\begin{tabular}{|l|l|}
\hline AÑO & TÍTULO DE LA PELÍCULA EN ESPAÑA \\
\hline 2000 & Erin Brockovich; Dinosaurio; La tormenta perfecta \\
\hline 2001 & - \\
\hline 2002 & La máquina del tiempo; Ice age: la edad de hielo \\
\hline 2003 & El núcleo; La tierra prometida \\
\hline 2004 & El día de mañana \\
\hline 2005 & Sahara \\
\hline 2006 & Ice age 2: el deshielo; Hoot: pequeños salvajes; Una verdad incómoda; Happy feet \\
\hline 2007 & $\begin{array}{l}\text { El corazón de la tierra; Sunshine; Los Simpson; Tierra; El último invierno; Michael } \\
\text { Clayton; Bee movie }\end{array}$ \\
\hline 2008 & $\begin{array}{l}\text { Paisajes transformados; Nuestro pan de cada día; Tres días; El incidente; Wall E; } \\
\text { Babylon A.D.; Encuentros en el fin del mundo; Cenizas del cielo; Nosotros } \\
\text { alimentamos el mundo; Quantum of solace; Ultimátum a la Tierra }\end{array}$ \\
\hline 2009 & La hora 11; Home; Ice age 3: el origen de los dinosaurios; Pandorum; 2012; Avatar \\
\hline 2010 & Teniente corrupto; La carretera; Océanos; En pata de guerra \\
\hline 2011 & $\begin{array}{l}\text { Las aventuras de Sammy; Objetivo Terrum; ¿Para qué sirve un oso?; Rebelión en la } \\
\text { isla; Happy feet 2 }\end{array}$ \\
\hline 2012 & $\begin{array}{l}\text { Una aventura extraordinaria; Lorax: en busca de la trúfula perdida; Soul surfer; Lo } \\
\text { imposible }\end{array}$ \\
\hline 2013 & $\begin{array}{l}\text { El corazón del roble; Bestias del sur salvaje; Tierra prometida; After Earth; Elysium; } \\
\text { Colonia V; Al final todos mueren; Rompenieves }\end{array}$ \\
\hline 2014 & Khumba; En el ojo de la tormenta; La sal de la Tierra; Interstellar \\
\hline 2015 & $\begin{array}{l}\text { Quédate conmigo; Autómata; Kingsman: servicio secreto; Mad Max: furia en la } \\
\text { carretera; Tomorrowland; Segundo origen; 45 años }\end{array}$ \\
\hline 2016 & $\begin{array}{l}\text { El infierno verde; Norman del norte; Mañana; Inferno; Before the flood; Marea } \\
\text { negra }\end{array}$ \\
\hline 2017 & $\begin{array}{l}\text { Psiconautas; Una verdad muy incómoda: ahora o nunca; Geostorm; Deep; Una vida a } \\
\text { lo grande }\end{array}$ \\
\hline 2018 & $\begin{array}{l}\text { Siete hermanas; Ready player one; Marea humana; Vengadores: infinity war; } \\
\text { Operación huracán; El reverendo; El Papa Francisco: un hombre de palabra; Venom; } \\
\text { Mortal engines }\end{array}$ \\
\hline La mujer de la montaña; Vengadores: endgame; Arctic dogs \\
\hline
\end{tabular}

